\title{
Evolución positiva en la satisfacción del alumnado en la Asignatura “Genómica” del Grado de Biotecnología tras la implementación de metodologías de aprendizaje activo.
}

\section{Santiago Vilanova y José Gadea}

Departamento de Biotecnología, Universitat Politècnica de Valéncia, Camino de Vera s/n 46022, Valéncia.jgadeav@ibmcp.upv.es sanvina@upvnet.upv.es

\begin{abstract}
The speed at which strategies and technologies are developed in Biotechnology makes mandatory a learning method focused on autonomous learning and practical cases. The objective of this study is to evaluate the evolution of the student's level of satisfaction regarding the learning strategies applied in the subject "Genomics" of the UPV Biotechnology Degree among two consecutive academic years. Several changes were introduced these years in project-based learning and flip-teaching.A substantial improvement in the level of satisfaction in these two strategies indicates the key aspects that have to be considered when applying flip-and project-based learning in Biotechnology.
\end{abstract}

Keywords: autonomous learning, flip-teaching, active learning, project, biotechnology

\footnotetext{
Resumen

La velocidad a la que las tecnologías y estrategias están cambiando in Biotecnología hace necesario un aprendizaje más enfocado a casos prácticos y aprendizaje autónomo. El objetivo de esta comunicación es evaluar la evolución en el grado de satisfacción de los estudiantes en referencia a los métodos docentes aplicados en la asignatura Genómica del Grado de Biotecnología de la UPV en dos años consecutivos. Varias modificaciones han sido introducidas en este curso académico. La sustancial mejora en el nivel de satisfacción obtenido indica sobre qué aspectos es necesario incidir para aplicar con éxito metologías como la clase inversa o el aprendizaje basado en proyectos.
}

Palabras clave: apendizaje autónomo, clase inversa, aprendizaje activo, proyectos, biotecnología 


\section{Introducción}

La Biotecnología es una disciplina donde debe implementarse el aprendizaje autónomo. La velocidad a la que tecnologías y estrategias experimentales cambian obliga a un aprendizaje muy diferente a la mera transmisión de conocimientos, que pueden quedarse obsoletos en un período de tiempo muy reducido. Este aprendizaje debe incluir la búsqueda activa de las posibilidades existentes para solucionar un determinado problema y la toma de decisiones para seleccionar una determinada estrategia de trabajo, sin tener la certeza de que sea la única manera de abordar dicho problema. También implica enfrentarse a situaciones de incertidumbre, a respuestas argumentadas, pero sin solución única, un escenario al que los estudiantes no están acostumbrados. Además, los proyectos biotecnológicos reales son multidisciplinares, por lo que el conocmiento en todos los ámbitos de un proyecto no exuste, y un aprendizaje cooperativo es imprescindible. Esta última situación mitiga la excesiva competitividad existente entre los alumnos de Biotecnología, lo que podría ser beneficioso para ellos a largo plazo.

Por otra parte, el aprendizaje autónomo implica la intervención del estudiante en el establecimiento de sus objetivos, procedimientos, recursos, evaluación y momentos de aprendizaje (Crispin et al, 2011). Estas estrategias confían en un aprendizaje activo, eliminando el sistema pasivo basado en clases magistrales, pone énfasis en un aprendizaje más profundo y un mejor entendimiento del tema tratado, aumenta la responsabilidad por parte del estudiante e incrementa su sentido de autonomía, y al mismo tiempo, proporciona un ambiente donde la relación profesor-alumno puede ser replanteada.

Entre las estrategias utilizadas para ejercitar el aprendizaje autónomo, en la asignatura Genómica del grado de Biotecnología utilizamos desde hace años una combinación de diseño de proyectos en equipos de estudiantes, y de clase estructurada en casos reales. El aprendizaje basado en proyectos es una metodología que permite a los alumnos adquirir los conocimientos y competencias clave en el siglo XXI mediante la elaboración de proyectos que dan respuesta a problemas de la vida real. El trabajo en equipos, por otra parte, refuerza otras competencias transversales, ya que actualmente las organizaciones requieren de personas que sepan administrarse dentro de los equipos de trabajo (Northwest Regional Educational Laboratory, 2006).

El año 2016-2017 introdujimos aprendizaje basado en clase inversa en nuestra asignatura, y presentamos los resultados de satisfacción del alumnado en las diferentes estrategias utilizadas en la asignatura (estructura general, tareas de clase inversa, proyectos). El resultado más destacable fue la elevada satisfacción por el proyecto de trabajo en grupo, así como la baja aceptación de la clase inversa. Esta año, presentamos los resultados comparados de dos cursos académicos consecutivos, y discutimos las mejoras obtenidas en relación a los ejercicios que se han implementado, lo que puede dar ideas sobre la implementación exitosa de la clase inversa.

\section{Objetivos}

Desde el curso académico 2012-2013, llevamos proponiendo diseños de proyectos en Genómica como ejercicio de aprendizaje autónomo a los estudiantes de Biotecnología de

(c)) BY-NC-ND 2018, Universitat Politècnica de València

Congreso IN-RED (2018) 
tercer curso de la Universidad Politécnica de Valencia. Los proyectos consisten en emular los diferentes pasos que un profesional en biotecnología deberá realizar al enfrentarse a un proyecto de estas características, búsqueda bibliográfica, selección de tecnologías, toma de decisiones, diseño experimental, localización de reactivos y equipamiento, presupuesto económico y cronograma de realización, basándose en cada punto en estudios similares que ya estén publicados en contextos diferentes. Con esto, los alumnos se enfrentan a la realidad de un proyecto de investigación (o un proyecto empresarial), que requiere no sólo un conocimiento teórico de un ámbito concreto de la Biotecnología, sino tener en cuenta muchos otros factores que condicionan el éxito real del proyecto.

Por otra parte, en el curso 2016-2017 iniciamos una estrategia docente de clase inversa, con tareas planteadas a los estudiantes, cuyo objetivo fue intentar que se consolidara la información teórica que los estudiantes reciben con clases magistrales distribuidas selectivamente a lo largo de la asignatura. Los estudiantes realizaban sobre todo tareas en Google Drive, alternando lectura de artículos científicos seleccionados, con visionado de videos o búsqueda de información en la web. Los resultados obtenidos en las encuestas de clase inversa nos forzaron a reestructurar las tareas, reduciendo el tiempo necesario en casa para realizarlas, ampliando plazos de entrega, y no abusando de la lectura completa de artículos científicos. Por tod ello, la presente comunicación pretende:

- Realizar una encuesta al alumnado para conocer el grado de satisfacción de la asignatura

- Evaluar la evolución en la estructura de la asignatura utilizando los datos de las encuestas de dos años consecutivos, teniendo en cuenta las mejoras explicadas en el documento.

\section{Desarrollo de la innovación}

\subsection{Encuestas de evaluación}

Mediante el uso de encuestas enfocadas a evaluar la experiencia de los estudiantes en esta iniciativa, se contactó a los estudiantes de Biotecnología del curso 2017-2018, y se elaboraron resultados que permiten la interpretación detallada. Las encuestas pretenden desvelar su grado de satisfacción con este tipo de docencia, la repercusión de este tipo de aprendizaje en el conocimiento de la asignatura, la originalidad y valor añadido de los mismos, relación profesor-alumno, etc. Las encuestas se realizaron online utilizando la herramienta de formularios de Goole Drive (https://www.google.com/intl/es_ALL/drive/).

La encuesta consta de 21 items con la siguiente escala de evaluación de Likert (Briones, 1995) : Muy en desacuerdo(1), En desacuerdo(2), Indiferente(3), De acuerdo(4) o Muy de acuerdo(5). Los items se subdividen en cuatro secciones: a) estructura general de la asignatura, b) la experiencia en la realización de casos prácticos, c) la experiencia en la realización de tarea de clase inversa, y d) la experiencia en la realización de trabajos/proyectos de investigación

El cuestionario fue el siguiente: 


\section{(EA) Estructura general de la asignatura}

EA1.Me ha resultado más EFECTIVA que la clase tradicional (profesor explicando y alumnos atendiendo) para entender los contenidos y su aplicación.

EA2. Me ha resultado más ENTRETENIDA que la clase tradicional (profesor explicando y alumnos atendiendo) para entender los contenidos y su aplicación.

EA3. Me ha ayudado a COMPRENDER contenidos en lugar de MEMORIZARLOS

EA4.Me ha ayudado a SABER APLICAR contenidos en lugar de MEMORIZARLOS

EA5. Hace que el profesor esté más implicado en la docencia, y por tanto interacciono más con él.

EA6. En un plan de estudios ideal sin tanta sobrecarga, recomendaría este tipo de docencia a otras asignaturas.

EA7. Respecto a las otras asignaturas con las que se ha compartido en el año ¿Has dedicado más o menos tiempo de estudio a GENOMICA?

\section{(CP) Casos Prácticos}

CP1. Me han ayudado a CONSOLIDAR los contenidos teóricos

CP2. Me han ayudado a ver cómo se APLICA la teoría en la realidad

CP3. Me han quitado el miedo a enfrentarme a artículos científicos

CP4. Me han confundido más que ayudado

\section{(T) Tareas}

T1. Me han servido de BASE para la materia que se iba a explicar en clase, si son anteriores, o de CONSOLIDACIÓN, si son posteriores

T2. Me han quitado mucho tiempo de estudio

T3. Siendo sincero/a...¿¿ué porcentaje de las tareas has contestado como si fuera a ser puntuada como una pregunta de examen?

\section{(P) Proyectos}

P1. Me han ayudado de discriminar entre información más relevante y menos relevante para mis objetivos

P2. Me han ayudado a darme cuenta de que puedo seguir adelante sin entender todos los detalles de un proyecto

P3. Me han ayudado a unir información proveniente de diferentes fuentes para resolver problemas específicos

P4. Me han ayudado a mejorar mis habilidades en estructurar la información escrita y oral

P5. Me han ayudado a mejorar mis habilidades en trabajar con otros en grupo

P6. Me han ayudado a mejorar mis habilidades en organizar y planificar mi tiempo

P7. Me ha motivado especialmente ver que soy capaz de crear un proyecto que nadie ha planteado hasta entonces.

\subsection{Análisis de datos}

(cc) EY-NC-ND 2018, Universitat Politècnica de València

Congreso IN-RED (2018) 
Los datos obtenidos a partir de las encuestas se analizaron mediante la aplicación hojas de calculo de Google Drive.

\section{Resultados}

Disponemos actualmente de más de 70 encuestas realizadas durante el curso anterior (Vilanova y Gadea, 2017), y de 34 encuestas delaño 2017-2018, con el objetivo de evaluar si la experiencia ha mejorado en dos años consecutivos. Para el año 2016-2017, representa el 70\% de los estudiantes matriculados este año, y para el año 2017-2018, el 40\%, por lo que consideramos la encuesta significativa. Para el año 2017-2018, un 38\% de los encuestados están matriculados en el grupo de Alto Rendimiento Académico del Grado en Biotecnología, y el resto en el grupo que se imparte en castellano.

A continuación pasamos a detallar los resultados más relevantes obtenidos en este proyecto:

\subsection{Estructura de la asignatura.}

Los estudiantes fueron preguntados si la estructura de la asignatura, de modo general (incluyendo tareas de clase inversa, clases magistrales alternas, y realización de trabajos de investigación, así como su división temática en casos, les ayudaba a comprender y/o a saber aplicar los contenidos en lugar de a memorizarlos, así como si este tipo de docencia les parecía más efectiva respecto a la docencia clásica de clase magistral, y si les parecía más entretenida. Finalmente, se les preguntaron aspectos generales como si recomendarían este tipo de docencia en un plan de estudios ideal sin tanta carga académica, y si habían dedicado más o menos tiempo de estudio a esta asignatura como consecuencia del tipo de docencia.

En el año académico 2016-2017, se observó que una no despreciable proporción del alumnado (42\%) se mostraba indiferente ante la efectividad de la asignatura, a pesar de que consideraban la estructura más entretenida (EA2 Fig1). Ese mismo año, se observó una tendencia positiva en cuanto a si el método docente les ha ayudado a comprender y saber aplicar mejor los conceptos de la asignatura (EA3 Fig 1. y EA4 Fig 1.). Un 52\% consideró positiva la experiencia, frente a un $30 \%$ que no ve diferencia entre este método y el aprendizaje clásico. Sin embargo, llamaba la atención el porcentaje de estudiantes que aún se decantan por un aprendizaje más clásico, ya que el 20\% consideraron esta estructura más inefectiva, y un 40\% no ven ninguna ventaja respecto al aprendizaje tradicional (EA1 Fig 1.). Recalcábamos que muchos de ellos podrían preferir un sistema de memorización pasiva, al que ya están habituados desde pequeños, y que les genera unos mejores rendimientos en cuanto a notas, frente a un nuevo sistema que les obliga a implicarse activamente en el aprendizaje.

Este año, las mejoras introducidas en la asignatura, sobre todo en la reestructuración de las tareas de clase inversa, así como en la mayor tutorización del trabajo de grupo, han provocado cambios positivos en las encuestas de satisfacción, como se observa en la Figura 1. Por una parte, destaca el alto porcentaje de alumnos que esta vez ha considerado la metodología más efectiva (70\%), sin detrimento de lo entretenida (67\%). Asimismo, el porcentaje de alumnos que consideran que la estructura les ha ayudado a comprender 
mejor, a saber aplicar, o a interaccionar con el profesor, ha aumentado sustancialmente (EA3, EA4 y EA5, respectivamente, con 63\%, 67\% y 67\% de estudiantes con valoración positiva o muy positiva, frente a $52 \%, 45 \%$ y $44 \%$ en el año anterior). Es de destacar que seguimos este año sin aumentar la carga que los estudiantes tienen que dedicar a esta asignatura, con un 67\% dedicándole el mismo tiempo o incluso menos que a otras asignaturas del año).
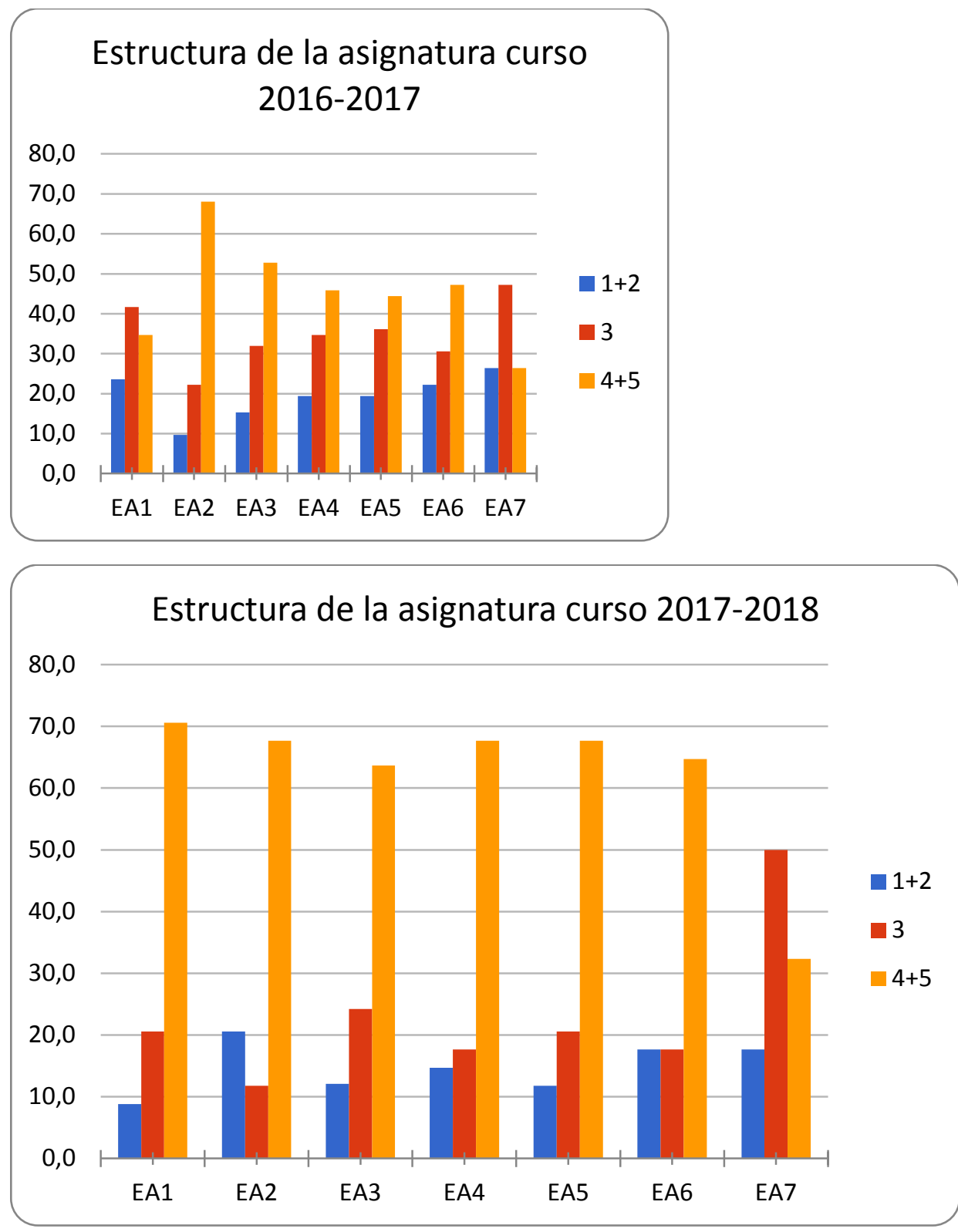

Fig. 1. Diagrama de barras que indica las puntuaciones de los items para la sección Estructura general de la asignatura en los años 2016-2017 y 2017-2018. Se han sumado las puntuaciones 1 y 2 que muestran disconformidad (Muy en desacuerdo y En desacuerdo) así como las puntuaciones 4 y 5 que muestran conformidad (De acuerdo y Muy de acuerdo). Los ítems EA1 a EA7 están detallados en el apartado 4.1

(cc) EY-NC-ND 2018, Universitat Politècnica de València 


\subsection{La estructura en casos prácticos}

Los estudiantes fueron preguntados, de nuevo, si una estructura en casos prácticos ayuda a comprender y saber aplicar los contenidos. Como dato muy positivo, (y similar a lo obtenido el año anterior para un 77\% de los estudiantes), un $85 \%$ de los encuestados este año piensa que este división las ayuda mucho a saber aplicar los contenidos (CP2 Fig. 2), ya que así pueden individualizar los conceptos y aplicarlos en casos tipo. Preguntados si con la lectura de artículos científicos (muchos casos plantean este tipo de ejercicios) habían conseguido perderle el miedo a lecturas de artículos, conseguimos nuestro objetivo de mejorar respecto al año anterior , con un 70\% con valoración positiva o muy positiva (CP3 Fig 2.). El año anterior, concluíamos que la lectura en un idioma extranjero (el $100 \%$ de los artículos propuestos están en inglés) podía ser una causa de este último porcentaje. Esta año, los artículos científicos seleccionados se entregan con texto resaltado en únicamente los párrafos y conceptos más relevantes para poder afrontar el caso práctico estudiado. De este modo, los estudiantes realizan una lectura “dirigida” y "simplificada” del artículo, lo que puede favorecer su comprensión. 


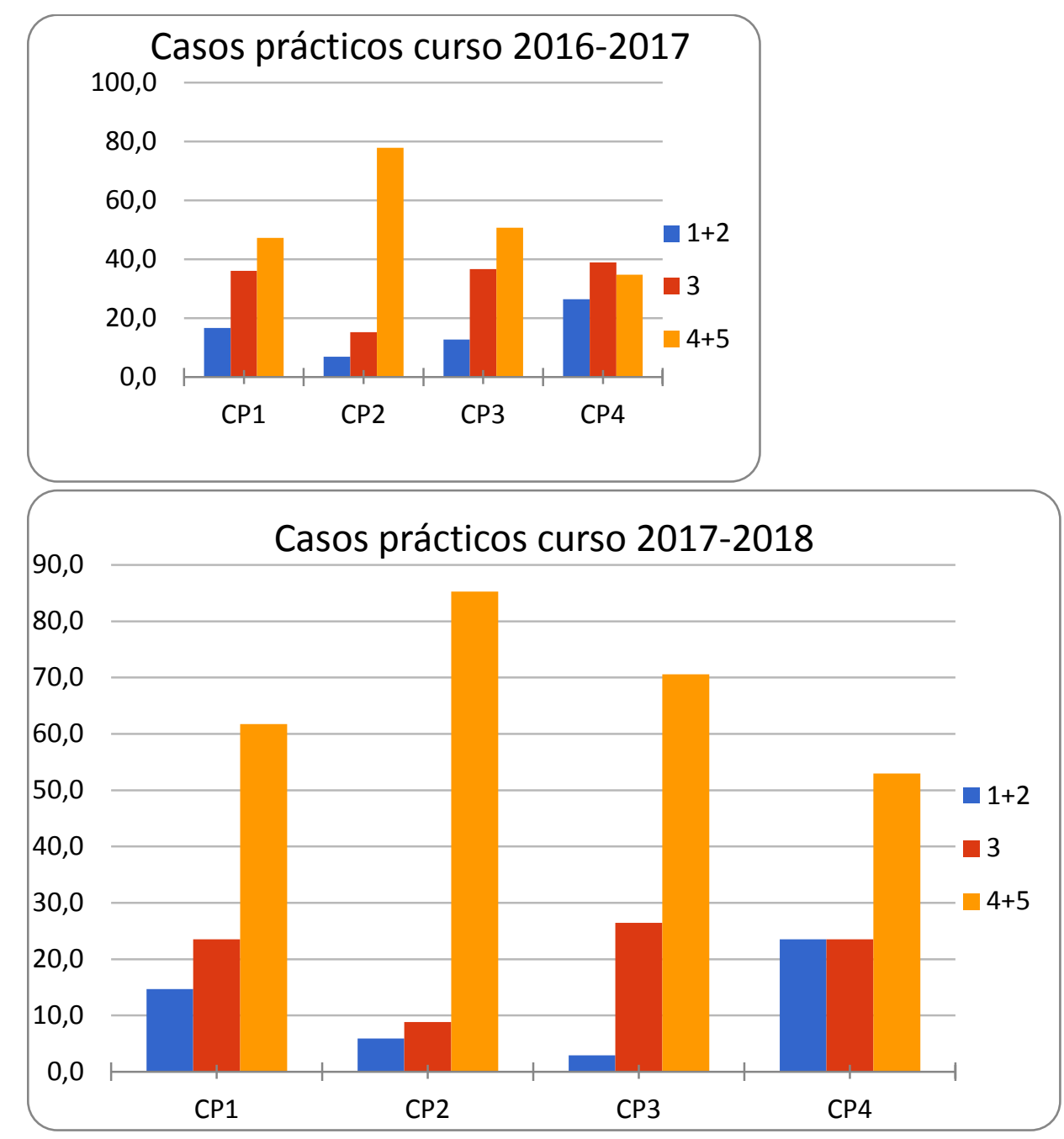

Fig. 2. Diagrama de barras que indica las puntuaciones de los items para la sección casos prácticos en los años 2016-2017 y 2017-2018. Se han sumado las puntuaciones 1 y 2 que muestran disconformidad (Muy en desacuerdo y En desacuerdo) así como las puntuaciones 4 y 5 que muestran conformidad (De acuerdo y Muy de acuerdo). Los ítems CP1 a CP4 están detallados en el apartado 4.2

\subsection{Las tareas en casa (La clase inversa).}

El año anterior, el punto más conflictivo de la encuesta lo encontramos en la implantación del sistema de clase inversa. Preguntados si este tipo de clases con tareas en casa, anteriores o posteriores a los conceptos temáticos, que sirvan de base, o ayuden a consolidar los contenidos, había sido útil, las respuestas siguieron una distribución normal centrada en el punto central (ni sí ni no), con un 30\%, un 27\% en contra y el resto más indiferentes(T1 Fig 3.). El año académico 2016-2017 fue es el primer año de la implantación en nuestra asignatura de la clase inversa, y quizá este resultado reflejaba las enormes posibilidades de mejora que teníamos todavía. Al mismo tiempo, un $48 \%$ de los estudiantes indicaron que la realización de estas tareas le había quitado mucho tiempo de estudio (T2 Fig 3.), lo que quizá se refleja en la calidad de las respuestas, ya que hasta un 35\% confiesa que no han

(cc) EY-NC-ND 2018, Universitat Politècnica de València 
respondido a las tareas como si fueran preguntas de examen, probablemente por la falta de tiempo en su realización(T3 Fig 3.). Este año, hemos ajustado las tareas en tiempo y cantidad (tiempo máximo de trabajo en casa 15-20 minutos, y sólo un par de tareas por caso) para evitar la saturación y conseguir una mayor implicación del alumno en las mismas. Según las encuestas de este año, estas modificaciones han resultado positivas, ya que observamos que frente a un $28 \%$ que no consideraba esta metodología nada útil (respuestas negativas o muy negativas) hemos reducido esta porcentaje hasta el 12\%, y esta reducción ha supuesto un aumento del número de valoraciones positivas (del 30\% el año pasado al $48 \%$ este año). La reducción del tiempo de las tareas se ve reflejada en la pregunta T2, donde un 56\% de los estudiantes no consideró que dichas tareas le quitaban tiempo de estudio, frente a sólo el 22\% el año anterior. La reducción del número de tareas y su simplificación ha hecho que los estudiantes respondan ahora con más seguridad, como refleja la pregunta T3.

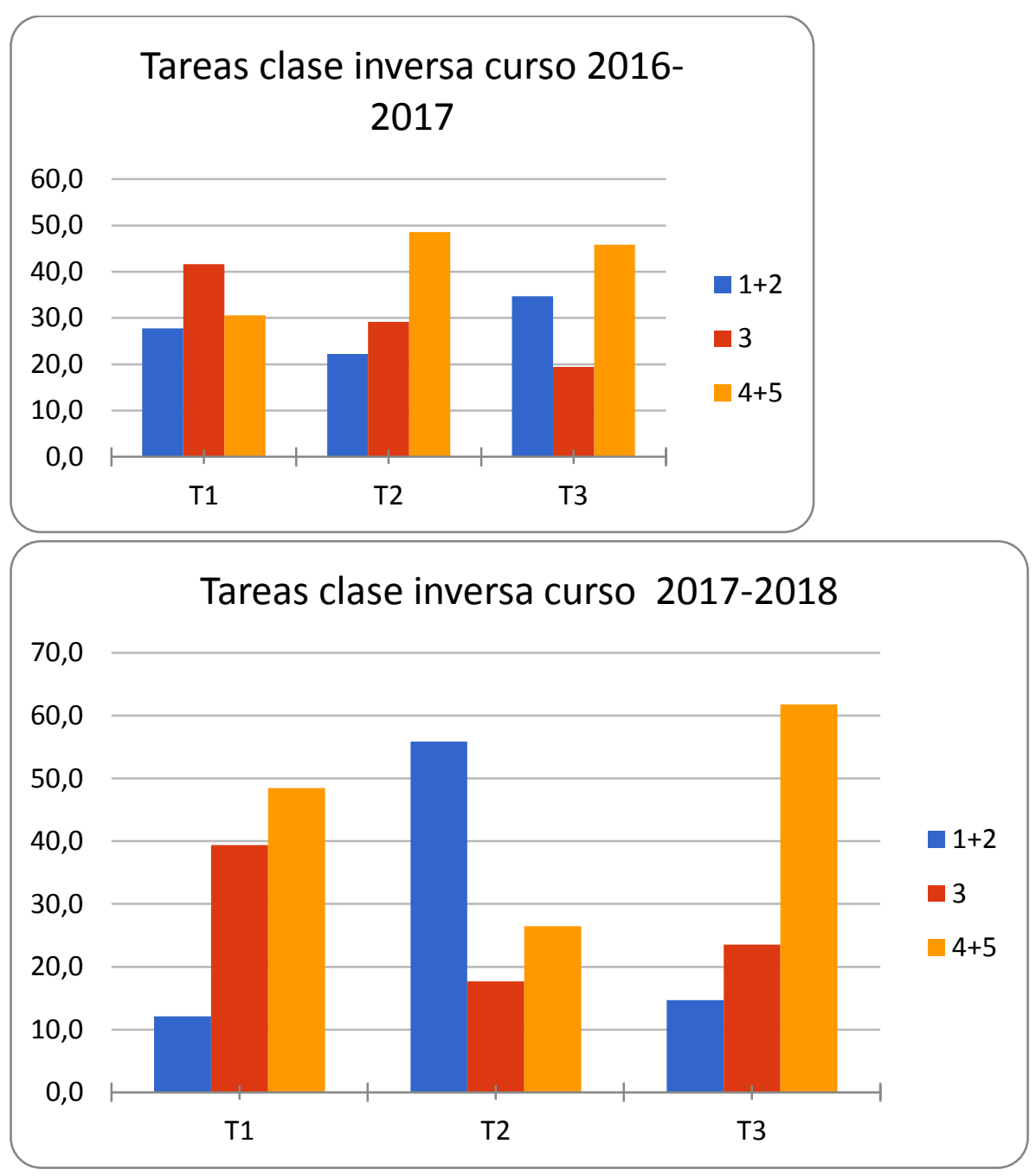

Fig. 3. Diagrama de barras que indica las puntuaciones de los items para la sección Tareas en casa. Se han sumado las puntuaciones 1 y 2 que muestran disconformidad (Muy en desacuerdo y En desacuerdo) así como las 
puntuaciones 4 y 5 que muestran conformidad (De acuerdo y Muy de acuerdo). Los ítems T1 a T3 están detallados en el apartado 3.1

\subsection{Los trabajos/proyectos de investigación}

Este punto sigue siendo el aspecto más positivo de la asignatura. Implantados desde el año 2012-2013, estos trabajos han supuesto desde hace años un modo de involucrar a los estudiantes en proyectos casi reales, y el feedback recibido durante estos años ha sido muy positivo. Este tipo de trabajos evalúa la Competencia Transversal "Diseño de Proyectos". Los estudiantes fueron preguntados por aspectos como los siguientes: si les ha ayudado a discriminar información relevante e irrelevante para un determinado objetivo (P1 Fig 4.), si les ha ayudado a entender que pueden seguir adelante sin entender todo un proceso (P2 Fig 4.), si les ha ayudado a buscar e integrar diferentes fuentes para crear algo nuevo (P3 Fig 4.), si les ha ayudado a trabajar en grupo y a planificar su tiempo (P4,P5 y P6 Fig 4.), y si les ha motivado crear algo nuevo desde cero (P7 Fig 4.).

Este año, hemos introducido una mayor tutorización en la realización de los mismos, lo que hasta la fecha se ha traducido en un mayor rendimiento académico, aunque la asignatura es anual y los resultados definitivos no se tendrán hasta el mes de junio.

La tendencia general hasta la fecha es muy positiva en todas las respuestas, y nos anima a seguir con esta iniciativa original que, que sepamos, no realiza ninguna otra asignatura de nuestro actual plan de estudios. Este año, los porcentajes de valoración positiva aumentan, con un 79\% que reconoce que la realización de estos trabajos le ha motivado, frente a sólo un $8 \%$ que lo ve negativamente, (P7 Fig 4.). Algunos porcentajes particularmente interesantes con tendencias al alza respecto al año anterior: Más de un $80 \%$ considera que les ha ayudado a discriminar información no relavante y a salir adelante sin entender la totalidad de un problema (P1 y P2 Fig 4.), o un 76\% que indica que le ha ayudado a encontrar fuentes diversas y crear algo nuevo (P3 Fig 4.), de nuevo porcentajes mejorados respecto al año anterior en aspectos importantes en la multidisciplinaridad de proyectos como los que se manejan en esta asignatura.

Finalmente, preguntados qué sentían mientras realizaban este proyecto, más de un 60\% contestó esta opción: "Nunca he olvidado que era una obligación, pero encantado/a de que me obliguen a cosas así!”., aumentando la cantidad de alumnos que disfrutan con esta actividad (frente a un 50\% del año anterior).

(cc) EY-NC-ND 2018, Universitat Politècnica de València

Congreso IN-RED (2018) 


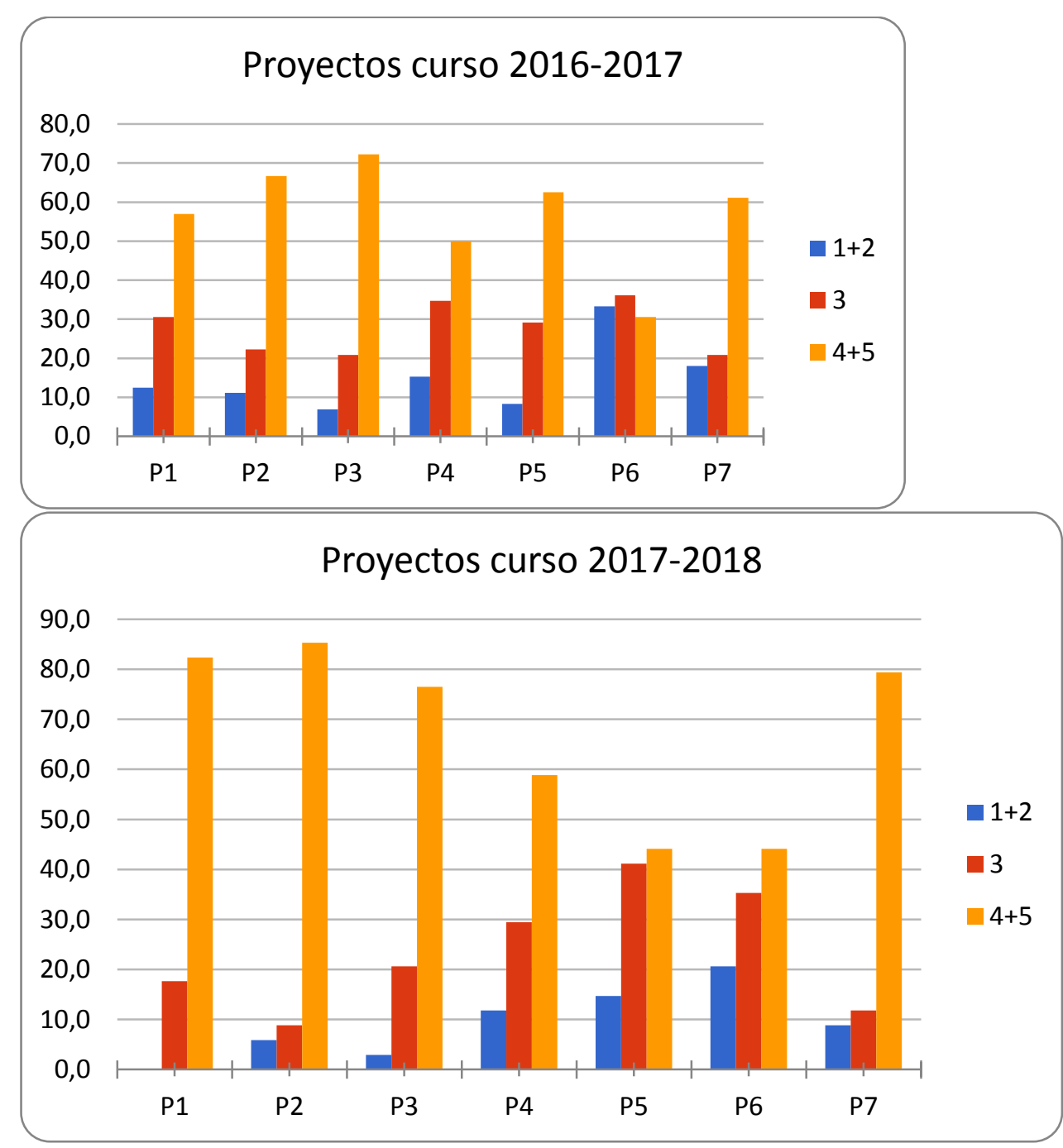

Fig. 4. Diagrama de barras que indica las puntuaciones de los items para la sección Proyecto de investigación para los cursos 2016-2017 y 2017-2018. Se han sumado las puntuaciones 1 y 2 que muestran disconformidad (Muy en desacuerdo y En desacuerdo) así como las puntuaciones 4 y 5 que muestran conformidad (De acuerdo y Muy de acuerdo). Los ítems P1 a P7 están detallados en el apartado 3.1

\section{Conclusiones}

Los resultados obtenidos este año académico, mejorados respecto a los buenos resultados del año anterior, nos siguen animando a continuar con el sistema docente que hemos implantado en esta asignatura de tercer curso del grado de Biotecnología. La reducción del tiempo de dedicación de los alumnos en casa para la realización de las tareas de clase inversa creemos que ha sido la clave de la mayor aceptación de las mismas, así como a reducción del número de tareas. Esto ha llevado a una mayor satisfacción general de la asignatura y a que sea considerada una asignatura "atractiva". Desde luego, quedan muchos aspectos susceptibles de mejora, pero demuestran que las metodologías de aprendizaje 
autónomo son una muy buena herramienta para el aprendizaje en Biotecnología. Los aspectos de la asignatura que ya parecen más enraizados y que según la encuesta siguen siendo una excelente iniciativa son los proyectos de investigación aplicada, implantados ya desde hace 6 años. Este año, la mayor tutorización de los estudiantes en la realización de los mismos ha contribuido a la mejora de unos ya elevados porcentajes de satisfacción respecto al año anterior.

En resumen, la implantación de estrategias de aprendizaje autónomo que ponga énfasis en un aprendizaje más aplicado, que aumente la responsabilidad por parte del estudiante e incremente su sentido de autonomía, es visto con buenos ojos por una alta proporción del alumnado en esta encuesta bianual, y debería ser implantado de un modo organizado en los planes docentes, sustituyendo o alternando con estrategias docentes más tradicionales.

\section{Referencias}

BRIONES, G. (1995). Métodos y Técnicas de Investigación para las Ciencias Sociales,2a reimp., Ed. Trillas, México.

CRISPIN BERNARDO, MARÍA LUISA, AND MA. DEL CARMEN DORIA SERRANO (2011). Aprendizaje autónomo orientaciones para la docencia. México: Universidad Iberoamericana. http://www.uia.mx/web/files/publicaciones/aprendizaje-autonomo.pdf.

NORTHWEST REGIONAL EDUCATIONAL LABORATORY (2016). Aprendizaje por proyectos. http://www.eduteka.org/AprendizajePorProyectos.php; ABP en Eduteka. [Consultado el 25 de Marzo de 2017].

GADEA, J y VILANOVA S. (2017) Evaluación de Diferentes Metodologías de Aprendizaje Activo desde el Punto de Vista del Estudiante en la Asignatura Genómica del Grado de Biotecnología. INRED-2017

(cc) EY-NC-ND 2018, Universitat Politècnica de València 